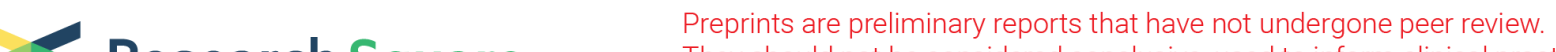 Research Square
or referenced by the media as validated information.
}

\section{Controlling the Twisted Intramolecular Charge- Transfer Character of Pyrene Derivatives Through Solvent Polarity}

\section{Young Mo Sung}

Samsung Advanced Institute of Technology

\section{Eun Suk Kwon}

Samsung Advanced Institute of Technology

\section{Yusuke Makida Maruyama}

Samsung Advanced Institute of Technology

\section{Youngsik Shin}

Samsung Advanced Institute of Technology

Soo-Ghang Ihn

Samsung Advanced Institute of Technology

Jong Soo Kim

Samsung Advanced Institute of Technology

Hyeonho Choi

Samsung Advanced Institute of Technology

Hyo Sug Lee

Samsung Advanced Institute of Technology

Jung-Hwa Kim

Samsung Advanced Institute of Technology Joonghyuk Kim

Samsung Advanced Institute of Technology

Soohwan Sul ( $\square$ soohwan.sul@samsung.com)

Samsung Advanced Institute of Technology

\section{Research Article}

Keywords: TICT, pyrene, solvatochromism, polarity, charge transfer, structural change

Posted Date: December 17th, 2021

DOI: https://doi.org/10.21203/rs.3.rs-1134592/v1 
License: (c) (i) This work is licensed under a Creative Commons Attribution 4.0 International License. Read Full License 


\section{Abstract}

Intramolecular charge transfer (ICT) plays a critical role in determining the photophysical properties of organic molecules, including their luminescence efficiencies. Twisted intramolecular charge transfer (TICT) is a process in which structural change accompanies ICT. Despite significant research, the relationship between TICT and solvent polarity, and its effects on photophysical properties, have been rarely investigated. Herein, we used time-resolved spectroscopy to study TICT in pyrene derivatives that are promising blue organic light emitting diode (OLED) emitter candidates; these derivatives show strong solvent-dependent charge-transfer (CT) behavior. Slight structural changes that do not affect excited state dynamics were observed in nonpolar solvents, while polar solvents were found to affect excited state dynamics and CT characteristics. The TICT behavior of these pyrene derivatives could be modulated through structural modification. Our study provides valuable guidelines for the control of optical properties, including the luminescence efficiencies of OLED emitters that show TICT characteristics.

\section{Introduction}

Charge transfer (CT) is a critical process in photosynthetic and metabolic mechanisms, ${ }^{1,2}$ and significant effort has been devoted to revealing the $\mathrm{CT}$ dynamics of various systems in nature, including chlorophyll. ${ }^{3-5}$ The recent advent of displays and photonic devices, such as organic light emitting diodes (OLEDs) and solar cells, has led to engineers as well as scientists devoting considerable attention to CT dynamics. ${ }^{6-8}$ Moreover, the spotlight has also been drawn to intramolecular charge transfer (ICT) in molecules having donor and acceptor moieties in a body due to their significant roles in determining emission efficiencies and orbital energy levels. ${ }^{9,10}$ ICT is often accompanied with structural changes that affect the electron-transfer mechanism; such a process is known as twisted intramolecular charge transfer (TICT). ${ }^{11}$ Because structural changes during the TICT process predominantly affect fluorescence efficiency, excited-state dynamics, and CT characteristics, a significant amount of research has focused on investigating CT behavior by controlling structures through changes in the viscosity and temperature of the medium. ${ }^{12-18}$ Compared to the abovementioned research foci, the effect of solvent polarity on the TICT process has been rarely investigated, despite the polarity of the solvent significantly perturbing CT dynamics by stabilizing or destabilizing the CT state, irrespective of any structural change. In this regard, we examined the effect of solvatochromism on TICT dynamics by synthesizing pyrene derivatives bearing carbazole and $\mathrm{N}, \mathrm{N}$-di(biphenyl) groups with and without $t$-butyl substituents and investigating their photophysical properties (Figure 1(a), detailed protocols for the syntheses of Pyr1-4 are provided in the Supporting Information).

Pyrene is an attractive material for electroluminescent devices due to its high fluorescence quantum yield and stability. ${ }^{19-21}$ The photophysical properties of pyrene can be controlled by substituents that alter its conjugation length and CT character. ${ }^{22-25}$ Pyrene derivatives substituted with electron donors/acceptors frequently exhibit CT characteristics without structural changes, and show absorption and emission 
behavior that depend on the polarity of the solvent. ${ }^{19-25}$ The pyrene derivatives (Pyr1-4) in this study have donor ( $N, N$-di(biphenyl))- $\pi$ (pyrene)-acceptor (carbazole) structures that exhibit CT characteristics and are also highly likely to show TICT because the $\mathrm{N}, \mathrm{N}$-di(biphenyl) group is well-known to undergo structural change in the excited state. ${ }^{12,26,27}$ In this regard, these pyrene derivatives are good candidates for revealing solvent-dependent TICT characteristics, particularly in terms of the photophysical properties.

\section{Results And Discussion}

The absorption spectra of the pyrene derivatives show almost identical spectral characteristics in six solvents with different polarities, with slight peak shifts and broadening (Figures 1(b) and S1). While the absorption spectra of Pyr1 and Pyr3 in hexane are very similar, those of Pyr2 and Pyr4 in hexane, while similar, show somewhat redshifted peaks. On the other hand, the absorption spectra are redshifted in the order: Pyr1 > Pyr2 > Pyr3 > Pyr4 in DMSO (Figure 1(d). This relationship between absorption spectral features and solvent polarity suggests that these pyrene derivatives exhibit different degrees of CT character in the ground state. To analysis this in more detail, we acquired fluorescence spectra of these pyrene derivatives, which show that fluorescence emissions are increasingly redshifted and broadened with increasing solvent polarity (Figures 1(c) and S2). As was observed for the absorption spectra, the fluorescence spectra of Pyr1 and Pyr3 in hexane are similar, while those of Pyr2 and Pyr4 in hexane have cognate spectra with somewhat redshifted peaks compared to those of Pyr1 and Pyr3. On the other hand, the fluorescence spectra of Pyr1 and Pyr2 show similar features in DMSO, while those of Pyr3 and Pyr4 have analogous spectra with somewhat blue-shifted peaks compared to those of Pyr1 and Pyr2 (Figure 1(e)). In other words, in the steady state results, Pyr1 (Pyr2) and Pyr3 (Pyr4) show analogous spectral features in the non-polar solvent, while Pyr1 (Pyr3) and Pyr2 (Pyr4) show spectral similarities in the polar solvent, which illustrates that the local excited (LE) states (in hexane) of these pyrene derivatives are affected by the $t$-butyl substituents, while their CT states (in DMSO) are mainly perturbed by the ortho/meta substituents on the $N, N$-di(biphenyl) group. The different steady-state trends observed in the non-polar and polar solvents imply that the molecular structures that might undergo TICT are solventpolarity dependent. Furthermore, the ortho/meta substituents on the $\mathrm{N}, \mathrm{N}$-di(biphenyl) group and the $t$ butyl substituents significantly affect structural change in the excited state.

To explore TICT in these pyrene derivatives, we calculated their ground- and excited-state molecular structures at the B3LYP/6-31G(d) (DFT) level of theory (Figure 2). The dihedral angles between the pyrene moieties and the $N, N$-di(biphenyl) substituents in these pyrene derivatives were all calculated to be around $50-70^{\circ}$ in the ground state, while those in the excited state are close to $90^{\circ}$. The orthogonal geometry endows the molecule with more distinct charge separation between the pyrene moiety and the substituent. ${ }^{26}$ Moreover, each $\mathrm{HOMO}$ is delocalized across the pyrene moiety and the $\mathrm{N}, \mathrm{N}$-di(biphenyl) substituent, while the LUMO is localized on the pyrene moiety, which facilitates CT from the $N, N$ di(biphenyl) substituent to the pyrene moiety (Figures S3-S7). Consequently, it is clear that these pyrene derivatives exhibit CT character accompanied by structural changes in their excited states (TICT), which 
is consistent with previous results that show that 4- $N, N$-dimethylaminobenzonitrile is planar in the ground state but has an orthogonal excited-state structure due to its TICT character. ${ }^{26,27}$

We used the Lippert-Mataga correlation, which provides information on the differences in the dipole moments of ground and excited states, to analysis more accurately on the CT states of our pyrene derivatives (Figure 3a). 28,29 The slopes of the Lippert-Mataga plots increase in the order: Pyr4 < Pyr3 < Pyr2 < Pyr1; Pyr1 (Pyr3) and Pyr2 (Pyr4) have almost identical slopes, while those of Pyr1 (Pyr2) and Pyr3 (Pyr4) are different, which indicates that the slope is significantly more affected by the ortho/meta substituents on the biphenyl rings of the $N, N$-di(biphenyl) moiety than the $t$-butyl substituents because the ortho/meta substituents severely hinder the ability to undergo structural change, which destabilizes the CT states. These results are supported by the calculated structures of the pyrene derivatives in their ground and excited states. The dihedral angles between the pyrene units and the $N, N$-di(biphenyl) groups in Pyr1 and Pyr2 are much closer to $90^{\circ}$ than those in Pyr3 and Pyr4 (Figure 2), which implies that the CT states of Pyr1 and Pyr2 are better stabilized than those of Pyr3 and Pyr4 because the orthogonal structure enhances charge separation by reducing orbital overlap between the pyrene moieties and the N,N-di(biphenyl) groups. ${ }^{26,27}$ In other words, the more-orthogonal excited-state structures of Pyr1 and Pyr2 stabilize the CT states and reduced their energy levels, in agreement with the lower slopes observed in the Lippert-Mataga plots of Pyr3 and Pyr4 compared to Pyr1 and Pyr2. Furthermore, these results also suggest that the ortho positions of the phenyl groups of the biphenyl substituent restricts structural change, while structural change is largely unaffected by the $t$-butyl substituents.

To elucidate details of the TICT mechanism operating in these pyrene derivatives, we measured their fluorescence quantum yields and lifetimes, which were observed to increase with increasing solvent polarity (Figures 3 and S8). Although longer fluorescence lifetimes are generally observed in polar solvents, higher quantum yields in polar media are uncommon because ICT processes typically increase non-radiative rates, resulting in lower quantum yields. In order to diagnose the lower quantum yields observed in nonpolar solvents, we determined the radiative and non-radiative rates of the pyrene derivatives (Figure 4 and Figure S9). The radiative rates tended to be lower in polar solvents (Figure S9), which is in good agreement with previous results. ${ }^{25,30-32}$ The non-radiative rates, however, were also lower in polar solvents, which is not generally observed for ICT processes. ${ }^{25,30-32}$ We exclude the possibility of dual emission (emission from both the LE and CT states) because the time-resolved emission spectra (TRES) of the pyrene derivatives show only single components that correspond to their fluorescence spectra (Figure S10).

We used transient absorption (TA) spectroscopy to rationalize the lower non-radiative rates in polar solvents. The TA spectra of the pyrene derivatives in hexane exhibit no spectral shifts in our experimental time window, while those in DMSO show significant blue-shift of the photo-induced absorption with increasing time (Figures 4(a,b), S11-S16). Considering the slow dynamics for CT formation (sub 10 ps) and the blue shifts of the induced absorption at the final time, we associate the fast components of the pyrene derivatives that are somewhat slower in polar solvents to CT formation accompanying structural 
change. ${ }^{12,17,18}$ Larger spectral shifts from the initial state to the final state were observed in polar solvents, which indicates that the CT states are better stabilized in polar solvents (Figures S11-S28). The TICT-based excited-state absorption shifts of Pyr1 and Pyr3 in the TA spectra are nearly identical to those of Pyr2 and Pyr4, respectively, which indicates that the CT states can be controlled by the substituents at the ortho/meta positions of the phenyl substituents on the $\mathrm{N}, \mathrm{N}$-di(biphenyl) group rather than the $t$-butyl substituents (Figure S29). A longer lifetime can be induced by a structural change that stabilizes the CT state in a polar solvent. The TICT rates determined from the TA data exhibit similar trends to the corresponding non-radiative rates (Figure $4(\mathrm{c})$ ). In other words, the non-radiative and TICT rates of the pyrene derivatives decrease with increasing solvent polarity, which indicates that TICT is the dominant process that determines the non-radiative rate and significantly affects the fluorescence quantum yield. Consequentially, the slow TICT process associated with a stabilized CT state slows the non-radiative process, which enhances the fluorescence quantum yield. Furthermore, the CT states of the pyrene derivatives are mainly stabilized by molecular torsional motion, which is much slower than a typical charge-separation process. This trend is opposite to that observed for general molecules that fluorescence less in more polar solvents. ${ }^{25,30-32}$

The TICT rates were analyzed in more detail by considering molecular structure. The pyrene derivatives in toluene, THF, and dichloromethane showed lower TICT rates (Pyr1 > Pyr2 > Pyr3 > Pyr4; Figures 4(c) and S11-S28), while this trend is different in high-polarity solvents (benzonitrile and DMSO: Pyr1 > Pyr3 > Pyr2 $>$ Pyr4), which indicates that insertion of the t-butyl group as well as the ortho substituents on the $\mathrm{N}, \mathrm{N}$ di(biphenyl) group reduces the rate of structural change during TICT in a polar solvent. ${ }^{33}$ Because much smaller structural changes are required to stabilize the CT states of the pyrene derivatives in non-polar solvents than in polar solvents, the effects of structural hindrance and the bulkiness imparted by $t$-butylgroup insertion and ortho substitution on the excited states are negligible in non-polar solvents.

Conversely, the CT states of the pyrene derivatives are stabilized in polar solvent through large structural changes; consequently, ortho substituents on the $N, N$-di(biphenyl) group and the $t$-butyl-group insertion significantly affects their CT states. In particular, the $t$-butyl substituents affect the TICT rates more than the ortho substituent, which implies that steric hindrance affects the TICT rate less than the bulkiness of the molecular structure. We rationalize this in terms of the bulkiness of the $t$-butyl substituents that increase the volume of the rotational groups in the TICT process, which leads to the slow TICT rates observed for Pyr2 and Pyr4. However, the steric hindrance of the $N, N$-di(biphenyl) group affects the TICT rate somewhat less, although it significantly destabilizes the CT state by hindering rotation. In other words, the CT rates are significantly affected by the bulkiness (volume) of the molecules rather than the steric hindrance associated with rotating groups, while the energy levels of the CT states are mainly affected by the steric hindrance of the rotating groups. To sum up, TICT processes in non-polar solvents are negligibly associated with structural change and are not significantly affected by modifications to the molecular structures. On the other hand, the TICT rates in polar solvents are controlled by the bulkiness of the $t$-butyl groups in these pyrene derivatives because structural changes are significantly affected by molecular volume. In addition, ortho/meta substituents on the $N, N$-di(biphenyl) groups of these pyrene 
derivatives negligibly affect CT rates because they hardly affect molecular volume; rather they stabilize the energy levels of the CT states.

\section{Conclusion}

In conclusion, we investigated how solvent polarity affects the TICT processes in a series of pyrene derivatives. These pyrene derivatives exhibit solvent-polarity-dependent photophysical properties. Structural changes hardly influence the excited state dynamics in hexane (non-polar solvent), while they significantly affect the excited state dynamics and perturb the CT states in the excited states in DMSO (polar solvent). TICT in these pyrene derivatives is controlled by the ortho/meta substituents on the $N, N$ di(biphenyl) group and the t-butyl substituents; the latter significantly influence the TICT rate, while the former mainly perturb the CT-state energy levels. This study provides guidelines for controlling the fluorescence efficiencies of pyrene derivatives that can be used in electroluminescent devices, such as OLED.

\section{Declarations}

\section{Notes}

The authors declare no competing financial interests.

\section{Acknowledgment}

The authors express their appreciation to Dong-Jin Yoon, and In-Sun Jung of Samsung Advanced Institute of Technology for their helpful advice in experiments and valuable discussions.

\section{References}

1. Mulliken, R. S. Molecular Compounds and Their Spectra. III. The Interaction of Electron Donors and Acceptors. J. Phys. Chem. 56, 801-822 (1952).

2. Mulliken, R. S. Molecular Compounds and Their Spectra. II. J. Am. Chem. Soc. 74, 811-824 (1952).

3. Novoderezhkin, V. I. \& van Grondelle, R. Physical Origins and Models of Energy Transfer in Photosynthetic Light-Harvesting Origins and Models of Energy Transfer in Photosynthetic Lightharvesting. Phys. Chem. Chem. Phys. 12, 7352-7365 (2010).

4. Moore, G. F. \& Brudvig, G. Energy Conversion in Photosynthesis: A Paradigm for Solar Fuel Production. Annu. Rev. Condens. Matter Phys. 2, 303-327 (2011).

5. Scholes, G. D.; Fleming, G. R.; Olaya-Castro, A. \& van Grondelle, R. Lessons from Nature About Solar Light Harvesting. Nat. Chem. 3, 763-774 (2011).

6. Brédas, J. L.; Norton, J. E.; Cornil, J. \& Coropceanu, V. Molecular Understanding of Organic Solar Cells: The Challenges. Acc. Chem. Res. 42, 1691-1699 (2009). 
7. Deotare, P. B. et al. Nanoscale Transport of Charge-Transfer States in Organic Donor-Acceptor Blends. Nat. Mater. 14, 1130-1134 (2015).

8. Brigeman, A. N. et al. Revealing the Full Charge Transfer State Absorption Spectrum of Organic Solar Cells. Adv. Energy Mater. 6, 1601001 (2016).

9. Eisenthal, K. B. Intermolecular and Intramolecular Excited State Charge Transfer. Laser Chem. 3, 145-162 (1983).

10. Kosower, E. M. Mechanism of Fast Intramolecular Electron-Transfer Reactions. J. Am. Chem. Soc. 107, 1114-1118 (1985).

11. Rettig, W. Charge Separation in Excited States of Decoupled Systems-TICT Compounds and Implications Regarding the Development of New Laser Dyes and the Primary Processes of Vision and Photosynthesis. Angew. Chem., Int. Ed. Engl. 25, 971-988 (1986).

12. Grabowski, Z. R.; Rotkiewicz, K. \& Rettig, W. Structural Changes Accompanying Intramolecular Electron Transfer: Focus on Twisted Intramolecular Charge-Transfer States and Structures Chem. Rev. 103, 3899-4032 (2003).

13. Fujiwara, T. et al. Electronic Spectra and Excited-state Dynamics of 4-Fluoro- $N, N$-dimethylaniline. Chem. Phys. Lett. 586, 70-75 (2013).

14. Pedone, A. Role of Solvent on Charge Transfer in 7-Aminocoumarin Dyes: New Hints from TD-CAMB3LYP and State Specific PCM Calculations. J. Chem. Theory Comput. 9, 4087-4096 (2013).

15. Maftei, D.; Zbancioc, G.; Humelnicu, I. \& Mangalagiu, I. Conformational Effects on the Lowest Excited States of Benzoyl-Pyrrolopyridazine: Insights from PCM Time-Dependent DFT. J. Phys. Chem. A 117, 3165-3175 (2013).

16. Lin, C.-K.; Wang, Y.-F.; Cheng, Y.-C. \& Yang, J.-S. Multisite Constrained Model of trans-4-(N,NDimethylamino)-4'-nitrostilbene for Structural Elucidation of Radiative and Nonradiative Excited States. J. Phys. Chem. A 117, 3158-3164 (2013).

17. Park, M.; Kim, C. H. \& Joo, T. Multifaceted Ultrafast Intramolecular Charge Transfer Dynamics of 4(Dimethylamino)benzonitrile (DMABN) J. Phys. Chem. A 117, 370-377 (2013).

18. Cha, W.-Y. et al. Two Modes of Photoinduced Twisted Intramolecular Charge Transfer in mesoArylaminated Subporphyrins. Chem. Commun. 50, 8491-8494 (2014).

19. Wiessner, A.; Hüttmann, G.; Kühnle, W. \& Staerk, H. Electron Transfer, Solvation, and Amplified Stimulated Emission of Pyrene-DMA and Anthracene-DMA. J. Phys. Chem. 99, 14923-14930 (1995).

20. Okada, T.; Migita, M.; Mataga, N.; Sakata, Y. \& Misumi, S. Picosecond Laser Spectroscopy of Intramolecular Heteroexcimer Systems. Time-Resolved Absorption Studies of $p-\left(\mathrm{CH}_{3}\right)_{2} \mathrm{NC}_{6} \mathrm{H}_{4}\left(\mathrm{CH}_{2}\right)_{\mathrm{n}^{-}}$ (1-pyrenyl) and -(9-anthryl) Systems. J. Am. Chem. Soc. 103, 4715-4720 (1981).

21. Dobkowski, J.; Rettig, W. \& Waluka, J. Intramolecular Charge-Transfer Properties of a Molecule with a Large Donor Group: The Case of 4'-(Pyren-1-yl)benzonitrile. Phys. Chem. Chem. Phys. 4, 4334-4339 (2002). 
22. Techert, S.; Schmatz, S.; Wiessner, A. \& Staerk, H. Photophysical Characteristics of Directly Linked Pyrene-Dimethylaniline Derivatives. J. Phys. Chem. A 104, 5700-5710 (2000).

23. Daub, J. et al. Competition between Conformational Relaxation and Intramolecular Electron Transfer within Phenothiazine-Pyrene Dyads. J. Phys. Chem. A 105, 5655-5665 (2001).

24. Weigel, W. et al. Dual Fluorescence of Phenyl and Biphenyl Substituted Pyrene Derivatives. J. Phys. Chem. A 107, 5941-5947 (2003).

25. Sung, J.; Kim, P.; Lee, Y. O.; Kim, J. S. \& Kim, D. Characterization of Ultrafast Intramolecular Charge Transfer Dynamics in Pyrenyl Derivatives: Systematic Change of the Number of Peripheral $\mathrm{N}, \mathrm{N}-$ Dimethyaniline Substituents. J. Phys. Chem. Lett. 2, 818-823 (2011).

26. Grabowski, Z. R. \& Dobkowski, J. Twisted Intramolecular Charge Transfer (TICT) Excited States: Energy and Molecular Structure. Pure Appl. Chem. 55, 245-252 (1983).

27. Sasaki, S.; Drummen, G. P. C. \& Konishi, G. Recent Advances in Twisted Intramolecular Charge Transfer (TICT) Fluorescence and Related Phenomena in Materials Chemistry. J. Mater. Chem. C 4, 2731-2743 (2016).

28. Lakowickz, J. R. Principles of Fluorescence Spectroscopy; Springer: New York, 208 (2006).

29. Mataga, N.; Kaifu, Y. \& Koizumi, M. Solvent Effects upon Fluorescence Spectra and the Dipolemoments of Excited Molecules. Bull. Chem. Soc. Jpn. 29, 465-470 (1956).

30. Kosower, E. M.; Dodiuk, H. \& Kanety, H. Intramolecular Donor-Acceptor Systems. 4. Solvent Effects on Radiative and Nonradiative Processes for the Charge-Transfer States of NArylaminonaphthalenesulfonates. J. Am. Chem. Soc. 100, 4179-4188 (1978).

31. Scarongella, M.; Laktionov, A.; Rothlisberger, U. \& Banerji, N. Charge Transfer Relaxation in DonorAcceptor Type Conjugated Materials. J. Mater. Chem. C1, 2308-2319 (2013).

32. Kim, W.; Sung, J.; Grzybowski, M.; Gryko, D. T. \& Kim, D. Modulation of Symmetry-Breaking Intramolecular Charge-Transfer Dynamics Assisted by Pendant Side Chains in $\pi$-Linkers in Quadrupolar Diketopyrrolopyrrole Derivatives. J. Phys. Chem. Lett. 7, 3060-3066 (2016).

33. Dahl, K.; Biswas, R.; Ito, N. \& Maroncelli, M. Solvent Dependence of the Spectra and Kinetics of Excited-State Charge Transfer in Three (Alkylamino)benzonitriles. J. Phys. Chem. B 109, 1563-1585 (2005).

\section{Figures}



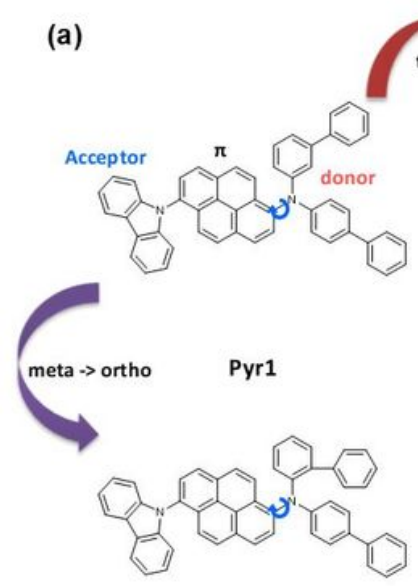

Pyr3 (b)

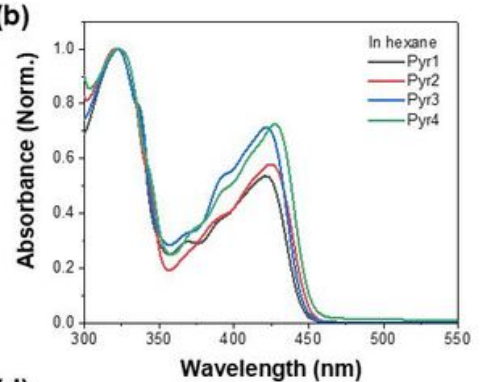

(d)

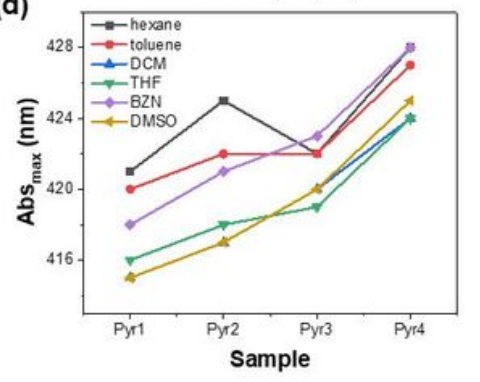

(c)

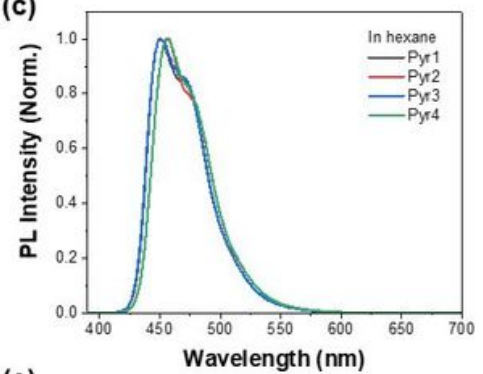

(e)

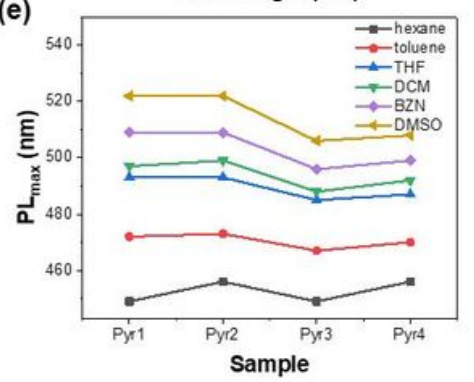

\section{Figure 1}

(a) Molecular structures of the pyrene derivatives in this study (Pyr1-4). Normalized (b) absorption and (c) fluorescence spectra of pyrene derivatives ((Pyr1-4) in hexane. Maximum (d) absorption and (e) fluorescence peak positions of the pyrene derivatives in various solvents (hexane, toluene, THF, dichloromethane (DCM), benzonitrile (BZN), and DMSO). 
(a)
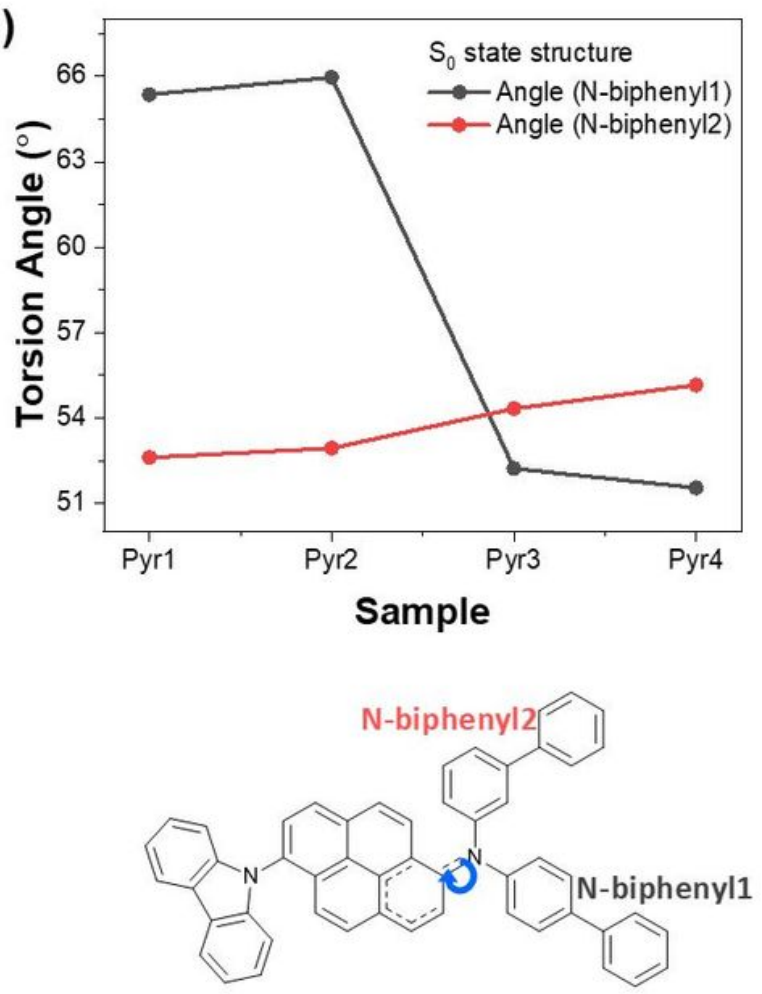

LE state, relatively planar structure (b)
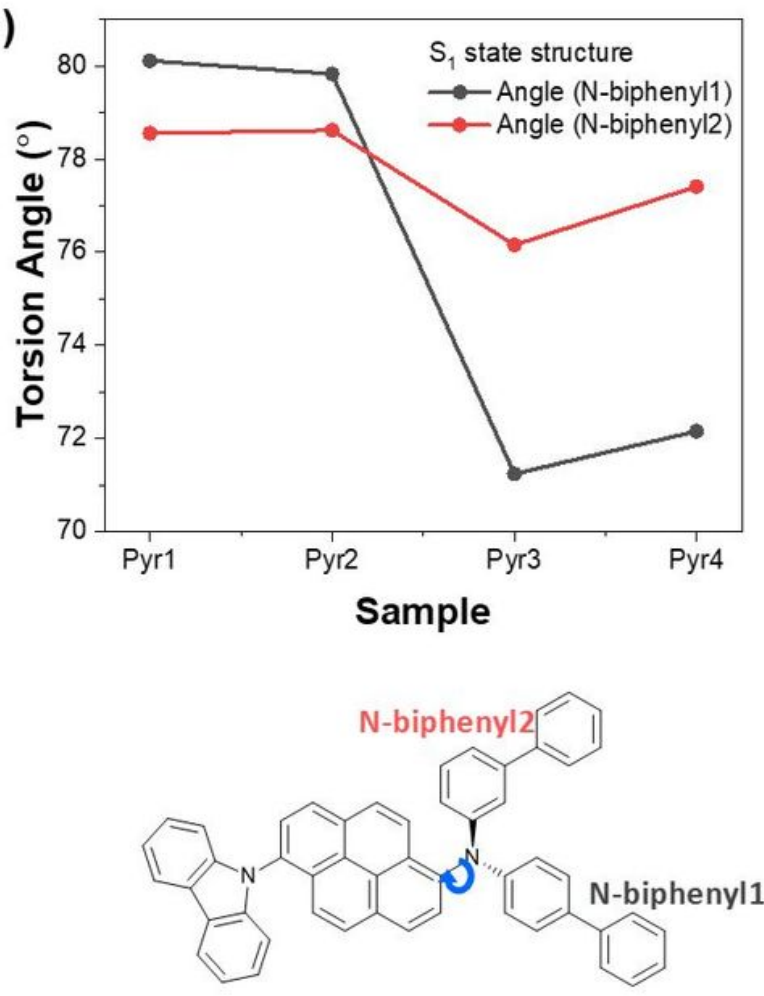

CT state, orthogonal structure

\section{Figure 2}

Torsion angles between the pyrene and biphenyl rings in the optimized (a) $S_{0}$ and (b) $S_{1}$ structures at the B3LYP/6-31G(d) (DFT) level of theory. 

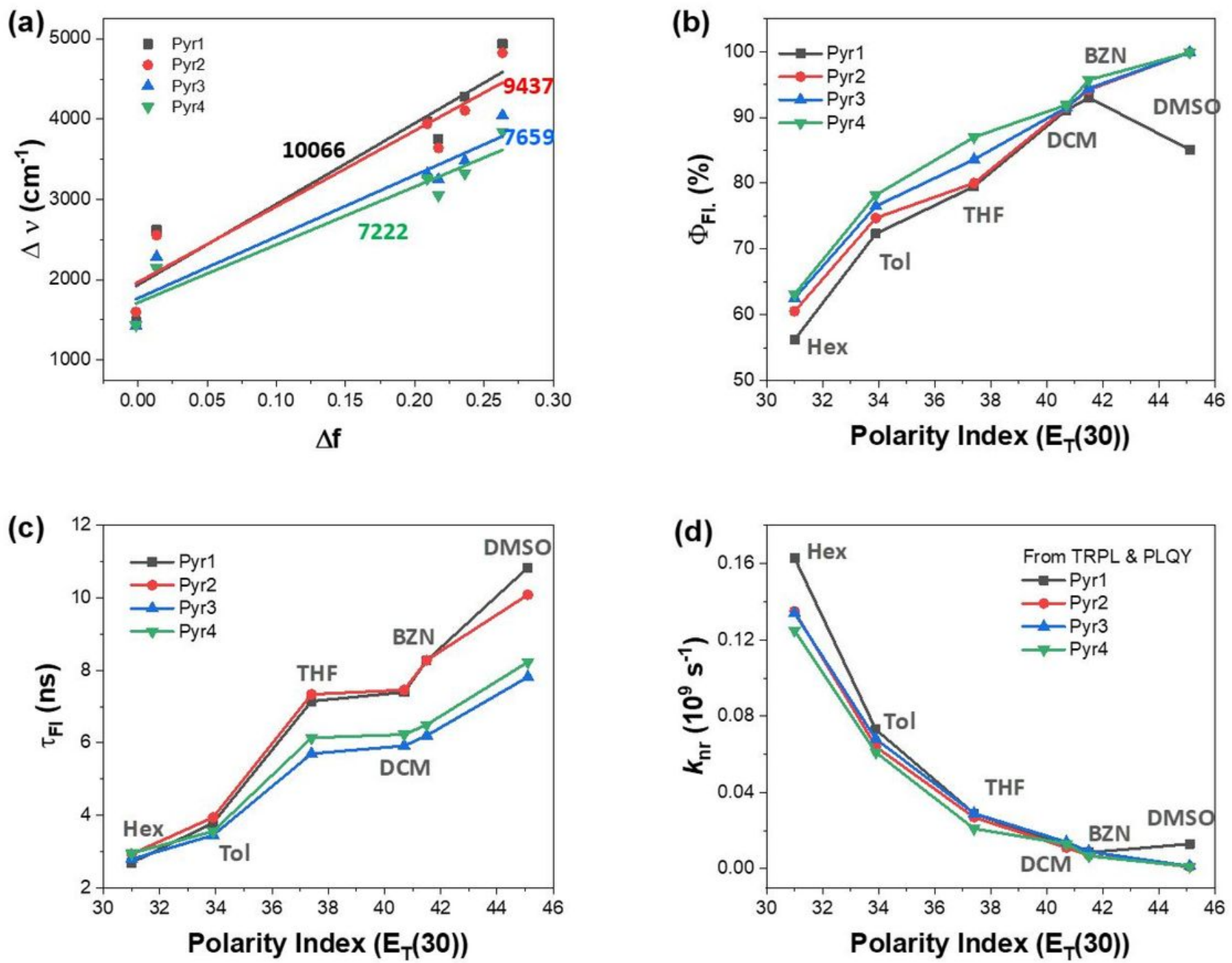

Figure 3

(a) Lippert-Mataga plot $\left(\Delta f=\left([(\varepsilon-1) /(2 \varepsilon+1)]-\left[\left(n^{2}-1\right) /\left(2 n^{2}+1\right)\right]\right)\right.$ for the pyrene derivatives. Fluorescence (b) quantum yields, (c) lifetimes, and (d) non-radiative-rates of the pyrene derivatives in various solvents (hexane, toluene, THF, dichloromethane (DCM), benzonitrile (BZN), and DMSO).
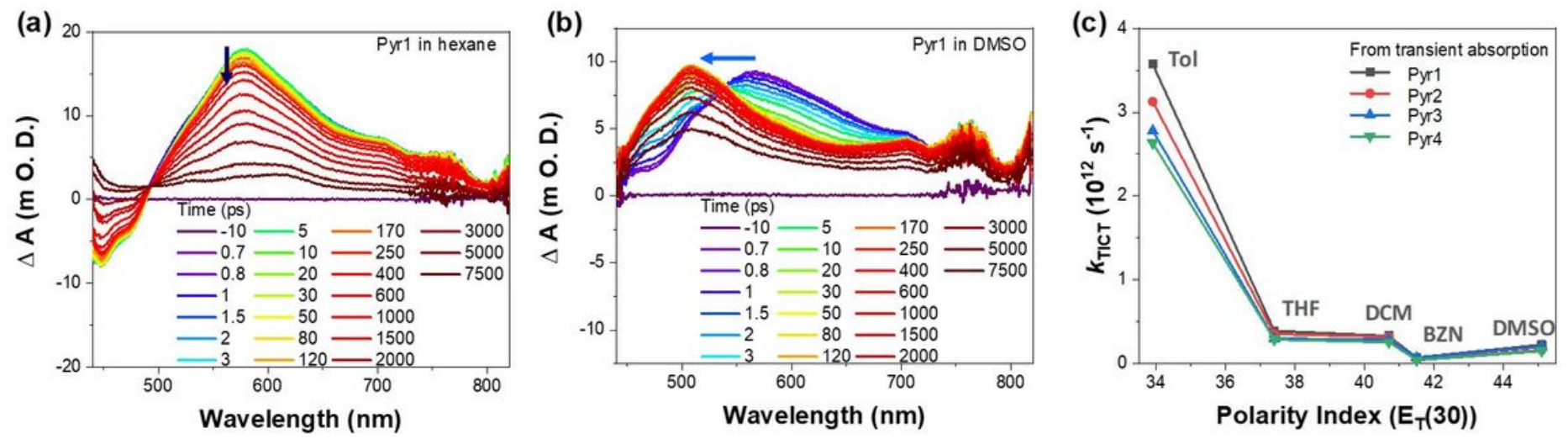
Figure 4

TA spectra of Pyr1 in (a) hexane and (b) DMSO. (c) TICT rates of the pyrene derivatives as functions of solvent polarity index $\left(\mathrm{E}_{\mathrm{T}}(30)\right)$.

\section{Supplementary Files}

This is a list of supplementary files associated with this preprint. Click to download.

- TOCGraphics.jpg 